\title{
Search for GRB counterparts
}

\author{
J. GREINER \\ Max-Planck-Institute for Extraterrestrial Physics, 85740 Garching, Germany
}

\section{INTRODUCTION - SEARCH STRATEGIES}

Historically, the search for $\gamma$-ray burst (GRB) counterparts began with the check of photographic exposures taken contemporaneously with the burst event ${ }^{1}$. Due to the lack of obvious associations of the GRB positions with well-known transients such as supernovae or flare stars, the search for correlations rapidly spread to very different energy ranges and detection methods. One example is the search for absorption events in the VLF $(164 \mathrm{kHz})$ propagation in the Earth ionosphere caused by the transit of (ionising) X-ray emission from the bursts ${ }^{2}$, which earlier proved successful for the detection of Sco X-1.

After these early (mid-seventies) attempts of simultaneous observations later on (eighties) the counterpart search was dominated by searches for non-correlated flaring as well as quiescent sources in different wavelength bands. This kind of investigation always used the implicit assumption that bursts repeat on timescales less than 50 years. Searches for archival optical transients in small GRB error boxes using large photographic plate collections were initiated at Harvard Observatory ${ }^{3}$ and then also performed at other observatories ${ }^{4,5}$. In the same manner, data of the Einstein , EXOSAT and ROSAT satellites were used to search for quiescent soft $\mathrm{X}$-ray sources ${ }^{6-10}$. In parallel, very few of the well-localised GRB error boxes of the second interplanetary network were examined with larger telescopes to search for faint quiescent optical counterparts ${ }^{11,12}$. No reliable counterpart candidate emerged from all these studies.

With the launch of the Compton Gamma-Ray Observatory (GRO) and the BATSE (Burst and Transient Source Experiment) measurements of about one burst per day improved possibilities emerged for the correlation with other (simultaneously performed) observing programmes. This includes the correlation of GRBs with (1) regularly exposed wide-field photographs of most major observatories ${ }^{13},(2)$ a CCDbased multi-camera monitoring of 0.75 ster of the sky ${ }^{14,15},(3)$ atmospheric airshower events due to $\mathrm{TeV}$ emission ${ }^{16-19}$, (4) neutrino or muon detection rates ${ }^{20-22}$. Due to an unexpected incident (the failure of the GRO tape recorders) and the advent of fast data transmission, rapid follow-up observations of GRB error boxes became possible with the development of BACODINE ${ }^{23}$ and the COMPTEL/BATSE/NMSU network $^{24}$. In the following I will concentrate on the new developments connected with these rapid follow-up observations as well as the availability of times and locations of many bursts for correlated investigations (see also other recent reviews ${ }^{25,26}$ ). 


\section{SIMULTANEOUS OBSERVATIONS}

\section{Monitoring with Dedicated Automated Telescopes}

The Explosive Transient Camera (ETC) is a wide (0.75 ster) field-of-view (FOV) CCD array consisting of 8 camera pairs which operates entirely by computer control on Kitt Peak National Observatory ${ }^{27}$. During its more than 4 years of operation there were five cases in which a BATSE GRB occurred during an ETC observation and within or near an ETC FOV ${ }^{14,15}$. No optical transients were detected during these observations, resulting in upper limits for the fluence ratio $\mathrm{L}_{\gamma} / \mathrm{L}_{\text {opt }} \geq$ $2-120^{14,15}$. Unfortunately, in all cases only a part $(20 \%-80 \%)$ of the rather large BATSE error box was covered. For those GRBs also seen by other satellites, triangulation will reduce the error box size and may increase the actual coverage.

\section{Photographic Sky Patrol}

For the correlation of BATSE GRBs and photographic wide-field plates a logistic network of 11 observatories with regular photographic observations has been established, including Sonneberg, Tautenburg and Hamburg (all FRG), Calar Alto (Spain), Ondřejov (CR), Odessa, Crimea (Ukraine), Dushanbe (Tadshikistan), Kiso (Japan), Australian UK and ESO (La Silla, Chile). For nearly 60 GRBs detected by BATSE since the launch of GRO simultaneous plates with typically $\mathrm{m}_{\text {lim }} \approx 2-3$ mag for a 1 sec duration flash have been identified ${ }^{13,28,29}$. No optical transient was found, resulting in limits for the flux ratio $\mathrm{F}_{\gamma} / \mathrm{F}_{\text {opt }} \geq 1-20^{13}$.

\section{Neutrino and Muon Fluxes}

Searches for neutrinos, anti-neutrinos and muons during times of BATSE GRBs have been performed using the data of the Irvine-Michigan-Brookhaven (IMB) detector $^{21}$, the Soudan 2 detector $^{20}$ and the Mont Blanc Neutrino Telescope ${ }^{22}$. Both samples are believed to contain primarily atmospheric events, i.e. neutrinos produced by the decay of secondary particles from cosmic-ray interactions in the Earths atmosphere. The IMB correlation with 183 Venera and Ginga GRBs revealed no neutrino coincidences. The resulting flux limit of $1.7 \times 10^{7}$ neutrinos $\mathrm{cm}^{-2}$ corresponds to $2.7 \times 10^{4} \mathrm{erg} / \mathrm{cm}^{2}$ for neutrinos in excess of $200 \mathrm{MeV}^{21}$. Also, no muon coincidences were found. A flux limit of $5.8 \times 10^{-7}$ muons $\mathrm{cm}^{-2}$ from neutrino interactions during GRBs is derived ${ }^{21}$.

A correlation of 180 BATSE GRBs with the Soudan 2 muon detector events revealed 18 trigger coincidences, near to the number expected by chance ${ }^{20}$. A correlation of the first 40 BATSE GRBs with events of the Mont Blanc telescope revealed no excess interactions. Depending on the neutrino flavour, the upper limits range between $2.5 \times 10^{10} \mathrm{~cm}^{-2} \mathrm{~s}^{-1}\left(\tilde{\nu}_{e}\right.$ at $\left.20 \leq \mathrm{E}_{\nu} \leq 50 \mathrm{MeV}\right)$ and $7.9 \times 10^{12} \mathrm{~cm}^{-2} \mathrm{~s}^{-1}\left(\tilde{\nu}_{\mu+\tau}\right.$ at $\left.20 \leq \mathrm{E}_{\nu} \leq 100 \mathrm{MeV}\right)^{22}$.

\section{TeV Emission}

A correlation of the 1991/1992 season 0.4-4 TeV events observed with the $10 \mathrm{~m}$ the Whipple Observatory Reflector with BATSE bursts has resulted in no positive 
detection which allows to set limits on the density of primordial black holes and the local density of cosmic strings ${ }^{19}$.

The CYGNUS-I air shower array detects ultra-high-energy (UHE) radiation at about 3.5 triggers/sec for $\mathrm{E}>50 \mathrm{TeV}$ from zenith and $\mathrm{E}>100 \mathrm{TeV}$ for $\theta>30^{\circ}$. For a total of 52 (out of 260) GRBs and 6 (out of 18) IPN GRBs having been in the CYGNUS FOV $\left(\theta<60^{\circ}\right)$ no evidence for UHE emission was found ${ }^{16}$. The limits for 3 GRBs are inconsistent with an extrapolation of the BATSE spectra, indicating either a softening of the production spectrum at high energies or the presence of UHE $\gamma$-ray absorption. If the latter possibility is true this would imply cosmological GRB distances ${ }^{16}$.

All GRBs of the BATSE 2B catalog with a zenith angle $<60^{\circ}$ and an location error $<15^{\circ}$ have been correlated with the HEGRA triggers. There are simultaneous data for 85 bursts but no significant excess could be found around the burst trigger times resulting in an upper limit between $10^{-7} \ldots 10^{-10} \mathrm{~cm}^{-2} \mathrm{~s}^{-1}$ for energies in the range of $40-500 \mathrm{TeV}^{17}$.

\section{NEAR-SIMULTANEOUS OBSERVATIONS}

\section{Monitoring Projects}

Due to the nature of the monitoring observations it is natural that the above discussed projects also allow searches for near-simultaneous coverage of burst positions. While simultaneous photographic exposures ${ }^{28}$ (12 hours before or after the burst) are available for dozens of GRBs with typical limiting magnitudes of $\mathrm{m}_{\text {lim }} \approx 7$ mag for 1 sec flash (and as short as about 1 hour after the burst), a recent ETC observation of GB 941014 happened to be only 150 sec after the burst onset. The 5 sec exposure with no optical transient sets a limit of $\mathrm{m}_{\text {lim }}=10.2 \mathrm{mag}^{15}$.

A HEGRA search in a time window of 12 hours after the bursts also revealed no significant excess rate ${ }^{17}$. This excludes strong extended $\mathrm{TeV}$ emission, as one could expect from the observation of the extended GeV emission from GB $940217^{30}$.

\section{Rapid Follow-up Observations}

BACODINE $^{23}$ (BATSE Coordinates Distribution Network) automatically evaluates the real-time telemetry stream from GRO, calculates approximate coordinates for a BATSE burst and distributes this position to interested observers. The COMPTEL/BATSE/NMSU network ${ }^{24}$ derives improved positions for those bursts occurring in the COMPTEL FOV using its imaging capabilities. The characteristics of both these systems are given in the Table.

\begin{tabular}{cccc}
\hline & BACODINE & COMPTEL/BATSE/NMSU & HETE \\
\hline Time delay & $5 \mathrm{sec}$ & $16 \mathrm{~min}$ & $5 \mathrm{sec}$ \\
Location error & $\pm 10^{\circ}$ & $\pm 1-2^{\circ}$ & $\pm 10^{\prime}$ \\
Rate & $20-50 \mathrm{yr}^{-1}$ & $2-5 \mathrm{yr}^{-1}$ & $5-20 \mathrm{yr}^{-1}$ \\
\hline
\end{tabular}


From the wealth of observational results I want to pick out only three examples, which should demonstrate the progress possible with the rapid burst notification:

(1) The Gamma Ray Optical Counterpart Search Experiment (GROCSE) located at Lawrence Livermore National Laboratory adapted a wide-FOV telescope with a total of 23 cameras with $7.7 \times 11.5$ FOV each for optical burst follow-up observations ${ }^{31}$. During $0.5 \mathrm{sec}$ integration time a limiting magnitude of $\mathrm{m}_{l i m} \approx 8$ mag is reached, and images are taken at a repetition rate of every 5 sec. During the first year of operation 8 BACODINE triggers were received and observations of the burst locations started for two of these events while the GRB was still bursting. For event 1 (2) the first image was taken 24 (17) sec after the onset of the burst which lasted 40 (90) $\sec ^{32}$. While the analysis of these events still is in progress, the limits achieved are considerably deeper than any previous observations.

(2) Within the observing campaign of GB 940301 by the BATSE/COMPTEL/ NMSU rapid response network the COMPTEL error box was observed seven hours after the burst with the $1 \mathrm{~m}$ Schmidt telescope at Socorro reaching a limiting magnitude of $\mathrm{m}_{V} \approx 16 \mathrm{mag}^{33}$. Still, no optical transient was found. Radio observations at the same time at $8.42 \mathrm{GHz}$ with the $34 \mathrm{~m}$ Goldstone antenna also found no new radio object ${ }^{33}$.

(3) The bright gamma-ray burst GB 940301 was monitored at $1.4 \mathrm{GHz}(2.6 \mathrm{FOV})$ and $0.4 \mathrm{GHz}\left(8^{\circ} .1 \mathrm{FOV}\right)$ at the Dominion Radio Astrophysical Observatory (DRAO) Synthesis Telescope starting starting 3 days after the burst ${ }^{34}$. A total of 245 radio sources were identified in the summed image in an intensity range between 0.8-110 mJy, but none was identified as a candidate for a flaring/fading radio counterpart based on variability analyses. The daily upper limits on the nondetection are $\approx 3.5$ mJy at $1.4 \mathrm{GHz}$ and $55 \mathrm{mJy}$ at $0.4 \mathrm{GHz}$ and constrain some fireball parameters for cosmological GRB models ${ }^{34}$.

\section{SUMMARY AND PROSPECTS}

Despite the increasing efforts in the search for GRB counterparts no convincing candidate has been identified yet. While the limits for simultaneous emission are still not yet deep enough, those for up to a few hours after the bursts are getting constraining for models which predict 1) predict strong and/or long-duration afterglows, or 2) simple extrapolations of the measured X-ray to $\gamma$-ray spectra to lower and higher energies.

Observational improvements in the very near future can be expected mainly from (1) the next generation device of GROCSE which will allow observations with a smaller FOV down to $\mathrm{m}_{\text {lim }} \approx 12-13 \mathrm{mag}$ for a $1 \mathrm{sec}$ flash ${ }^{32}$, and (2) the launch of $\mathrm{HETE}^{35}$ which will measure burst positions with better than $10^{\prime}$ accuracy (see the Table) and after subsequent data transmission to ground observers will allow observations with smaller FOV telescopes (and thus fainter limiting magnitudes)

than feasible up to now. This for the first time will allow optical observations with a sensitivity which is comparable to the flux estimates deduced by extrapolating burst spectra down from the X-ray/ $\gamma$-ray range. 


\section{ACKNOWLEDGEMENTS}

I'm grateful to H.-S. Park, S. Barthelmy, T. Harrison and R. Vanderspek for communicating their results prior to publication. JG is supported by the Deutsche Agentur für Raumfahrtangelegenheiten (DARA) GmbH under contract FKZ 50 OR 9201.

\section{REFERENCES}

1. Grindlay J.E., Wright E.L., McCrosky R.E., 1974. ApJ 192, L113

2. Kasturirangan K., Rao, U., Sharma, D. et al. 1974. Nat. 252, 113

3. Schaefer B.E., Bradt, H., Barat, C., et al. 1984. ApJ 286, L1

4. Hudec R., Borovicka, J., Wenzel, et al. 1987. A\&A 175, 71

5. Greiner J., Flohrer J., Wenzel W., Lehmann T., 1987. ApSS 138, 155-171

6. Pizzichini G., Gottardi, M., Atteia, J., et al. 1986. ApJ 301, 641

7. Boër M., Hurley, K., Pizzichini, G., et al. 1991. A\&A 249, 118

8. Greiner J., Boër M., Motch C., et al. 1991. 22nd ICRC Dublin, vol. 1: 53-56

9. Greiner J., et al. 1995. NATO ASI C450, eds. M.A. Alpar et al. Kluwer, p. 519-522

10. Boër M., Greiner J., Kahabka P., et al. , 1993. A\&A Suppl. 97, 69

11. Vrba F., Hartmann D.H., Jennings M.C., 1995. ApJ (in press)

12. Sokolov V., et al. 1995. Flares and Flashes, eds. J. Greiner et al. , LNP (in press)

13. Greiner J., et al. 1994. Gamma-Ray Bursts, eds. G.J. Fishman et al. , AIP 307: 408-412.

14. Krimm H. et al. 1994. Gamma-Ray Bursts, eds. G.J. Fishman et al., AIP 307: 423-427.

15. Vanderspek R. et al. 1995. Flares and Flashes, eds. J. Greiner et al., LNP (in press)

16. Alexandreas D.E., Allen G.E., Berley D., et al. (CYGNUS Collab.), 1994. ApJ 426, L1

17. Matheis V., 1995. Ph.D. thesis, Heidelberg University

18. Aglietta M. et al. (EASTOP Collab.) 1993. 23rd ICRC, Calgary 1993, vol. 1: 61

19. Connaughton V. et al. 1994. Gamma-Ray Bursts, eds. G.J. Fishman et al., AIP 307: 470

20. DeMuth D.M. et al. 1994. Gamma-Ray Bursts, eds. G.J. Fishman et al. , AIP 307: 475

21. LoSecco J.M., 1994. ApJ 425, 217

22. Aglietta M., Antonioli P., Badino G., et al. 1993, 23rd ICRC, Calgary 1993. vol. 1: 69

23. Barthelmy S. et al. 1994. Gamma-Ray Bursts, eds. G.J. Fishman et al. , AIP 307: 643

24. Kippen R.M., Ryan J., Connors A. et al., 1995. this volume

25. Schaefer B.E., 1994. Gamma-Ray Bursts, eds. G.J. Fishman et al. , AIP 307: 382-391.

26. Hartmann D.H., 1995. Flares and Flashes, eds. J. Greiner et al., LNP (in press)

27. Vanderspek R. et al. 1994. Gamma-Ray Bursts, eds. G.J. Fishman et al., AIP 307: 438

28. Greiner J., et al. 1995. Flares and Flashes, eds. J. Greiner et al. , LNP (in press)

29. Hudec R., 1995. Flares and Flashes, eds. J. Greiner et al. , LNP (in press)

30. Hurley K., Dingus B.L., Mukherjee R., et al. 1994. Nat. 372, 652-654

31. Akerlof C. et al. 1994. Gamma-Ray Bursts, eds. G.J. Fishman et al., AIP 307: 633-637.

32. Park H.-S., 1994. AAS HEAD meeting, Napa Valley, Nov. 1994

33. Harrison T.E., McNamara B.J., Pedersen H., et al. 1995. A\&A (in press)

34. Frail D.A., Kulkarni S.R., Hurley K.C., et al. 1995. ApJ 437, L43-L46

35. Ricker G. et al. 1988. Nuclear Spectroscopy of Astrophysical Sources, eds. N. Gehrels et al. , AIP 170: 407 